\title{
Object Therapy: A Student-selected Component Exploring the Potential of Museum Object Handling as an Enrichment Activity for Patients in Hospital
}

\author{
Helen J Chatterjee (Corresponding author) \\ Research Department of Genetics, Environment and Evolution \\ School of Medical and Life Sciences and UCL Museums \& Collections \\ University College London, Gower Street, London, UK, WC1E 6BT \\ Tel: 44-207-679-4113 E-mail: h.chatterjee@ucl.ac.uk \\ Guy Noble \\ UCLH Arts, University College London Hospitals NHS Foundation Trust \\ ICT Directorate, 3rd Floor, Maple House \\ 149 Tottenham Court Road, London, UK, W1T 7BN \\ Tel: 44-845-155-5000 Ext: $5451 \quad$ E-mail: guy.noble@uclh.nhs.uk
}

\begin{abstract}
This study involved innovative research in a novel field, namely 'object therapy', within the framework of a student-selected component (SSC) undertaken by second year, Phase 1 Medicine students at University College London. The project had a series of intrinsic aims: to provide medical students with communication skills, methods of assessing wellbeing and research techniques and to evaluate the potential of museum object handling as an enrichment activity in hospitals. Five medical students undertook museum object handling activities at 24 patient's bedsides in order to assess the psychological impact of 'object therapy' using standard Quality of Life (QoL) measures. Quantitative analyses indicated an increase in overall well being and patient's perception of their health status at the end of the session. Qualitative analyses revealed patients felt positive about the role of object handling sessions as a distraction from everyday ward life. The study showed that this is a novel enrichment activity which has the potential to enhance patient's lives whilst in hospital. The SSC provided valuable communication and research skills to students in their pre-clinical year, affording an opportunity to interact with patients, gain ward experience and an appreciation of the importance of considering the whole person when treating a patient.
\end{abstract}

Keywords: Object therapy, Wellbeing, Quality of life, Museums, Enrichment

\section{Introduction}

Student-selected components (SSCs) are defined by the UK's General Medical Council (GMC) as that part of the undergraduate medical curriculum which goes beyond the limits of the core and allows students to study in depth in areas of particular interest to them, providing them with insights into scientific methods and the discipline of research that engenders an approach to medicine that is questioning and self-critical (GMC, 2003). At University College London (UCL) SSCs are a compulsory component for Phase 1 first and second year undergraduate Medicine students who are free to select a research project of their choosing. SSC co-ordinators at UCL encourage students to take advantage of the fact that the Medical School is part of a multi-faculty University and this is reflected in the wide range of SSCs offered, including non-biomedical science topics such as arts and humanities, fine art and languages.

Previous research has highlighted the importance of introducing innovation and originality into SSCs and suggested that SSCs provide opportunities for students to develop lifelong and transferable skills (Macnaughton, 1997; Yates et al., 2002). In this study we offered a Phase $12^{\text {nd }}$ year SSC entitled 'The role of museum loan boxes and object handling as an enrichment activity at University College Hospital'. As part of the module, students delivered one-to-one handling 
sessions at patients' bedsides using museum objects from UCL Museums \& Collections. The key goal of this research was to assess the value and potential of a closer engagement between heritage and hospitals based around museum object handling as an enrichment activity and in so doing "engender an approach to medicine that is questioning and self-critical" (GMC 2003, p. 40). The SSC fulfilled the necessary criteria for research innovation and originality through focusing on a topic not previously explored in a scholarly context. Some museums and libraries do offer services, such as loan boxes (a collection of museum objects which are available as an outreach resource) which are accessible by hospitals, but to date there has been no critical assessment of the impact of such sessions. The module required the students to enhance and develop a range of skills including critical and creative thinking, data analysis and collection (involving quantitative and qualitative techniques), scientific writing and multi-modal forms of communication. Finally, the involvement of museum collections in the research afforded new skills in object handling and raised student's awareness of how the Arts and the environment can impact upon patient wellbeing.

The project had a series of overarching aims including:

- To provide medical students with communication skills, methods of assessing wellbeing and research techniques

- To undertake an innovative project to demonstrate the unique, interdisciplinary, role museum collections can play in university teaching and research

- To evaluate the potential of museum object handling as an enrichment activity in hospitals

- To widen access to UCL Museums \& Collections to a new audience

- To widen the understanding of the role that the Arts and environment can play in patient care

The project thus constituted a multidimensional research study exploring the outcomes for both students (pedagogical) and patients (enrichment).

\subsection{Background}

The clinical basis for this research lies in the fundamental idea that improving the quality of the hospital environment and enriching patient's lives whist in hospital can have a positive effect for patients and staff. This was perhaps first recorded by Florence Nightingale in 1859 who noted that:

"The effect in sickness of beautiful objects, and especially of brilliancy of colour is hardly at all appreciated [by the authorities]...[The] variety of form and brilliance of colour in the objects presented to patients are actual means of recovery. " (Nightingale, 1859).

Some of the first quantitative clinical evidence came from Professor Roger Ulrich whose 1984 paper demonstrated that patients with a view of nature (trees) from their beds had a reduced length of stay and a slightly reduced need for analgesics in hospital compared to those with a view of a brick wall (Ulrich, 1984). Since then further clinical evidence has shown that arts in health interventions have several positive therapeutic and medical outcomes for patients including: reduction of stress, reduction of depression and anxiety, reduced blood pressure, reduced pain intensity, reduced need for medication, improved mental health and reduced length of stay (Staricoff, 2006). These studies have also shown that there are positive effects for staff and benefits in the education and training of medical practitioners including: improved morale and job satisfaction and consequently job retention, increased communication and awareness between patients and staff, reduced stress levels and increased observational skills (Staricoff, 2006; Dolev, et al., 2001). Furthermore, Macnaughton has recently argued that art in hospitals may have a wider role than purely providing a therapeutic environment and that hospitals may be being used as a cultural resource (Macnaughton, 2007).

This research has arisen from the proliferation of arts and health activity across all sections of health care. One such project, the partner to UCL in this research, is UCLH Arts, based at University College Hospitals NHS Foundation Trust. UCLH Arts is committed to providing a welcoming, uplifting environment for all patients, visitors and staff through the use of a varied and stimulating arts programme and in so doing aims to improve patient well being, boost staff morale and widen access to the Arts.

UCLH Arts achieves this in a number of ways including curating exhibitions, hosting music and drama events and partnering with museums and galleries to bring their collections to patient's and staff within the hospital. Often this takes the form of enrichment activity which in this case is defined as an activity which improves and or increases wellbeing. UCLH Arts aim to increase patient well-being and essentially enrich patients' lives using heritage and the Arts. Examples of this include the project presently discussed as well as providing live music by patients' bedsides and commissioning artists to work collaboratively with patients.

To date there is nothing that the authors could identify in the research literature directly relating to the role of, and potential, of museum object handling as an enrichment activity in healthcare. There are however several examples of museums extending their outreach education programmes to hospitals, care homes and other social welfare organisations (Arigho, 2008; Chatterjee, 2008; O'Sullivan, 2008; Noble and Chatterjee, 2008; Jacques, 2007). To this 
end we coin the term 'object therapy' to mean the inclusion of museum collections in enrichment and intervention activities in hospitals and other healthcare settings.

\section{Methods}

\subsection{Project plan}

The SSC was supervised by the authors (HC and GN) and prior to student recruitment permission was obtained to undertake the research from the UCLH Research Ethics Committee (REC) (MREC No 06/Q0505/78). The project ran from November 2006 to March 2007 and was timetabled as eight sessions each lasting up to three hours, with students encouraged to undertake reading and other preparation in their own private study time. The SSC was assessed via written work and general performance. The allocation of the work was broken down accordingly:

- $10 \%$ for the development of enrichment resources (including patient information leaflets and promotional flyers used to recruit patients)

- $10 \%$ for the development of suitable data collection methods (including patient evaluation forms and questionnaire design)

- An interim progress report (which carried no marks, but was compulsory)

- $10 \%$ for conceptual hypotheses, outreach and other skills (including patient communication skills)

- 70\% for a final written report of between 3,000 and 5,000 words (including a comprehensive literature review, materials, methods, data analysis, interpretation and future recommendations)

\subsection{Student training}

In 2006 five Year 2 Phase 1 medical students from UCL opted to undertake research into the role of heritage in hospitals. The project began with a series of training sessions which covered the following areas:

- An introduction to the role of arts in health

- Methods of assessing wellbeing and quality of life

- Museum object handling and museum outreach session delivery

- Patient communication skills, privacy and dignity in care, and patient confidentiality

- Infection Control

- Research skills

The students were also supplied with a reading list which included literature from the arts in health sector, museum object handling and museum education. In addition the students were given information about UCL Museums \& Collections (UCL Museums \& Collections, 2006), the history of UCL and object-specific information about the objects in their loan boxes. With training complete the students were inducted into University College Hospital's (UCH) volunteer programme which included training in infection control, patient confidentiality and ward logistics.

\subsection{Materials}

Each student was supplied with a 'loan box' which contained between 6 and 8 objects from UCL Museums \& Collections (www.museums.ucl.ac.uk). These boxes were lightweight metal photographic camera cases approximately $46 \mathrm{~cm} \times 32 \mathrm{~cm} \times 15 \mathrm{~cm}$ in size, which ensured the objects could be transported safely and easily. The boxes were lined with specialist museum packaging materials and each object was carefully wrapped in acid free tissue paper. Each box contained a range of objects including natural history specimens, archaeological artefacts and artworks. In addition each box contained a laying-out mat (a piece of object-friendly plastezote) which was used to protect the objects during the object handling session. Each student was also supplied with a digital audio recorder for the collection of qualitative information during object handling sessions, alcohol hand gel and an I.D badge.

An outline research protocol was developed as part of the REC application by the project supervisors; this was later refined in a group session with the students as part of their initial training. In his capacity as UCLH Arts Curator, GN approached staff from a variety of wards at $\mathrm{UCH}$ and recruited three wards to participate in the project. Following guidelines from the REC, and with input from the supervisors, the students developed a patient information leaflet (PIF) and consent form $(\mathrm{CF})$.

\subsection{Research Protocol}

Students delivered one to one object handling sessions at patients' bedsides using the museum loan boxes. As defined in the PIF, participation on the part of the patients was entirely voluntary. Patients were recruited by the students with assistance from the ward staff who often directed students towards those patients who were fit enough to participate (many patients on the participating wards were too unwell to be involved). Following patient recruitment the students fully explained the aims of the research, their role in delivering the object handling session and the research protocol 
which included the use of a digital voice recorder. At the start of each session the patient signed the CF and the students laid out all of the objects from the loan box on the patient's table, the audio recorder was switched on and the session could begin.

\subsection{Object handling sessions}

The research protocol comprised a series of introductory quantitative quality of life questions including two visual analogue scales (Figure 1), one of which assessed patient's perception of their overall health status and the other described patient's perceptions of their general well being; this was based on the EQ VAS design (EuroQol Group, 1990). These questionnaires also included space for the students to list basic patient information such as age and gender, and assign a research number. In addition patients' were asked to complete a mood adjective checklist (Table 1) the latter was loosely based on Watson, Clark and Tellegen's (1988) Positive Affect Negative Affect Scale (PANAS).

Once the quality of life questionnaires and mood checklist were completed the students asked each patient to choose an object and then a series of questions were posed, such as:

- Why have you chosen that object?

- What do you think this object is?

- What does the object feel like?

- Can you think of any experience that might relate to this object?

- Where do you think it comes from?

- What material do you think it is made out of?

Each session lasted between 10 to 20 minutes during which the patients were encouraged to handle and explore all of the objects. At the end of the object handling session the quantitative quality of life questionnaires and mood checklist were again completed by patients, the audio recorder was switched off and both parties either washed their hands or used alcohol gel.

\section{Results}

\subsection{Formative assessment with students}

As part of the introduction to the SSC and prior to the formal training, the authors led a group formative assessment with the students to explore their existing knowledge of arts in health, their feelings towards enrichment and intervention in healthcare and their reason for selecting the SSC. The students revealed that they had relatively limited knowledge of arts programming in hospitals but stated that their reason for choosing the SSC was to gain a better understanding of hospital enrichment activities and its benefits, to conduct interdisciplinary research and gain new skills (two students specifically cited a desire to increase patient communication skills), to learn more about museums and to work with colleagues across UCL and UCH.

\subsection{Data analysis}

The students made a combined total of 35 visits to UCH and interviewed 24 patients; patients suffered from a variety of illnesses, almost most were suffering from respiratory disorders, and were over 60 years of age. The length of hospital stays varied from 1 week to over 4 weeks. Post-data collection the students transcribed the audio-recordings and used basic mind- mapping methods to ascertain themes and trends in the qualitative information retrieved from these recordings. This technique involves the selection of key phrases and themes from interview transcripts, and mapping their frequency in a given data set. Quantitative quality of life data extracted from the visual analogue scales (VAS) were analysed using simple statistical techniques (i.e. Students' t-test) to understand frequency distribution.

Due to limited time and difficulties in recruiting patients the students decided to pool their interview transcripts, visual analogue and mood adjective data.

\subsection{Research outcomes}

Despite the fact that the students pooled their transcripts and data each student analysed these data independently. Table 2 highlights the key themes extracted using mind mapping by one student (PL) from patient interview transcripts. These results indicate that the most frequent theme expressed was a general interest and/or enjoyment of the object handling session. More patients expressed boredom or a lack of interest in the session compared to those who commented that the session suppressed boredom and an appreciation of enrichment activities. Few patients (only 8 out of 24) commented on their illness or made reference to pain during sessions. Other students described difficulties in mapping the frequency of themes using this technique citing vast differences in the range of topics explored in sessions. All of the students commented that most patients appeared to feel positive about the concept of handling objects but agreed that several patients stated that the objects presented were not of interest to them. The students all made reference to 
comments made by several patients relating to the sessions being a positive distraction from the stress and bustle of ward life and one patient who actually cited the session as a cause of decrease in their pain intensity.

Natural history objects (fossils, minerals and shells) were the most commonly selected objects with the brass etching plates, plus accompanying prints, and the archaeological pot shards, being the least favoured objects.

Two of the students (CA and DH) employed t-tests to analyse the VAS data. These tests showed that there was an overall improvement in patient's perception of their general wellbeing and health status when the VASs from the start of the handling sessions ('before') were compared to those from the end of the sessions ('after'). These results were significant at the $95 \%$ confidence level. Other quantitative analyses undertaken by the students indicated $57 \%$ of patients revealed a difference 'before' compared to 'after' the handling session with regard to general wellbeing, with $43 \%$ showing no difference 'before' compared to 'after'; $38 \%$ of patients revealed a difference 'before' and 'after' the handling session in health status perception, compared to $62 \%$ who showed no difference. None of the VAS data showed a decrease in either score after the session.

\subsection{Student outcomes: Summative assessment with students}

A summative assessment was carried out by the authors at the end of the project when the students submitted their write-ups. These discussions revealed mixed responses from the students with regard to the role of museum object handling as an enrichment activity in hospitals. Most of the students still felt positive about the role of arts in health and were generally enthusiastic about the use of museum collections in enrichment, aside from one student who expressed concerns that such activities may distract from clinical treatments. During the summative discussions the students also revealed certain challenges, notably difficulties in recruiting patients. Partly this difficulty related to the fact that wards are very busy and patients were often receiving treatment or visitors, but the students also cited cautiousness in approaching and communicating with certain patients, particularly those who were very ill.

\section{Discussion}

The research outcomes from the SSC indicate this is a novel enrichment activity which has the potential to enhance patient's lives whilst in hospital. Quantitative analyses indicated an increase in overall well being and patient's perception of their health status at the end of the session. Qualitative analyses revealed patients felt positive about the role of object handling sessions as a distraction from everyday ward life and had a positive impact on relationships amongst staff and patients. As well as providing evidence of object handling as a means of distraction, some patients also appeared to use the sessions as a tool for providing meaning to their illness and current health status. Such discussions could have valuable health benefits and a means for patients to find positive meaning in their illness, which Romanoff and Thompson (2006) state can lead to less depression and better quality of life and emotional wellbeing.

Crucially more research is needed to fully characterise this new engagement between heritage and hospitals and to thoroughly explore the therapeutic potential of object therapy for patients, their family and friends, carers, medical staff and students. This type of engagement with patients may be more appropriate for longer stay patients, such as those in chronic care facilities, hospices and rehabilitation units, where it could be used to stimulate patients, relive boredom and facilitate communication between caregivers and patients. Furthermore, in line with Macnaughton's (2007) recent study, the wider impact of arts programmes in hospitals and the role of hospitals as cultural resources warrants further investigation.

One concern which was repeatedly raised by the students throughout the project was the need to adequately control any negative emotions which may emerge during object handling sessions. The possibility of patients revealing negative memories, and showing distress and depression during sessions was discussed throughout the training period and the students were given guidance on how to handle these situations should they arise, including on site consultation with ward staff if the students felt sufficiently concerned (although this was never needed).

Student communication skills turned out to be an important factor which impacted both the length of the sessions and the students' effectiveness in engaging patients in the project. The summative assessment revealed that the students felt hindered by their lack of general communication skills and this was evident when we listened to the audio recordings which were often stilted and sometimes not overly enthusiastic on the part of the students. During training we impressed upon the students the need to employ a sense of excitement and wonder whilst trying to engage patients, but the students general lack of experience and perhaps confidence in talking openly with older people did seem to have impacted their delivery of sessions. One student in particular highlighted concerns over "not knowing how to talk to older people, especially when they are very ill". Notwithstanding this all of the students felt that the SSC had enhanced their patient communication skills and importantly given them first hand experience of everyday ward life in their pre-clinical year. In addition the experience encouraged them to think creatively about patient care and the importance of considering the whole person when treating a patient. It also emphasised the need for regular non-clinical communication training for medical students taking them out of their comfort zone and challenging them to work with patients in new ways. 
Reassuringly one of the key concerns expressed by the medical students was the lack of a control group with which to compare the results against. During the project we discussed the possibility of control groups and what form these should take. One idea was the inclusion of sessions which did not allow direct object handling but only visual inspection of the objects, but the main issue was what would constitute an effective control (i.e. the use of quantitative quality of life questionnaires in the absence of handling sessions? One-one interviews in the absence of any museum object-related activity?); a collective decision could not be reached on this point and hence the lack of any controls in the research.

\subsection{Future research and Conclusions}

This study has demonstrated that offering a novel SSC can have a range of positive benefits for students, staff and patients. Research at UCH using loan boxes from UCL Museums \& Collections is continuing to explore the potential of object handling as a therapeutic, enrichment and/or intervention activity (Chatterjee, 2008). This research has involved a variety of different session leaders including other students (MA Museums Studies students from UCL's Institute of Archaeology and the University of Toronto) and research nurses (UCL Institute for Women's Health). These studies continue to provide support for the positive benefits of providing access to museum collections in arts programming within hospitals and its valuable role in student and staff professional development, particularly with regard to the acquisition of communication and research skills. Future research, funded by the Arts and Humanities Research Council (Award no: AH/G000506/1), will explore the psychological impact of 'object therapy' in a longitudinal, multidisciplinary, study involving a range of researchers from UCL, ULH, several others hospitals and numerous partner museums.

\section{Acknowledgements}

We are extremely grateful to the five medical students who elected to undertake the SSC (Christopher Anthony, Iryna Bouberiak, Charles Gallaher, Deniz Hassan, Philippa Leighton), all of the patients at UCLH who participated in the research, ward staff on T 7, 9 and 10 at UCH for all their help and support, Jonathan Webster (Consultant Nurse for Older People at UCH), Celine West (Head of Learning and Access, UCL Museums \& Collections) and Caroline Selai (UCL Institute of Neurology).

\section{References}

Arigho, B. (2008). Getting a Handle on the Past: The Use of Objects in Reminiscence Work, in H. J. Chatterjee (ed) Touch in Museums: Policy and Practice in Object Handling, 205-12, Berg: Oxford.

Chatterjee, H. J. (2008). Touch in Museums: Policy and Practice in Object Handling. Oxford: Berg.

Dolev, J.C., Friedlander, L.K., Braverman, I. (2001). Use of fine art to enhance visual diagnostic skills. Journal of the American Medical Association, 286(9), 1020-1021.

EuroQol Group. (1990). EuroQol: A new facility for the measurement of health-related quality of life. Health Policy, 16, 199-208.

General Medical Council. (2003). Tomorrow's Doctors. London: GMC.

Jacques, C. (2007). Easing the transition: using Museums Objects with Elderly People, in E. Pye (ed) The Power of Touch: Handling Objects in Museum and Heritage Contexts, 153-161, Walnut Creek: Left Coast Publishing.

Macnaughton, R.J. (1997). Special study modules: An opportunity not to be missed. Medical Education, 31, 49-51.

Macnaughton, J. (2007). Art in Hospital Spaces: The role of hospitals in an aestheticised society. International Journal of Cultural Policy, 13(1), 85-101.

Nightingale, F. (1970[1859]). Notes on Nursing: What It Is, and What It Is Not. New Impression Ed. New York: Dover Publications.

Noble, G. and Chatterjee, H. J. (2008). Enrichment programs in hospitals: Using museum loan boxes in University College London Hospital, in H. J. Chatterjee (ed) Touch in Museums: Policy and Practice in Object Handling, 215-23, Oxford: Berg.

Romanoff, B.D. \& Thompson, B.E. (2006). Meaning Construction in Palliative Care: The use of Narrative, Ritual, and the Expressive Arts. American Journal of Hospice and Palliative Medicine, 23 (4) 309-16.

Staricoff, R. L. (2006). Arts in health: a review of the medical literature. Arts Council, Research Report, Vol 36.

O’Sullivan, J. (2008). See, Touch and Enjoy: Newham University Hospital's Nostalgia Room, in H. J. Chatterjee (ed) Touch in Museums: Policy and Practice in Object Handling, 224-230, Oxford: Berg.

UCL Museums \& Collections. (2006). Inspire: Highlights of UCL's Collections. London: Grant Museum of Zoology Press. 
Ulrich, R. S. (1984). View through a window may influence recovery from surgery. Science, 224, 42-421.

Watson, D., Clark, L. \& Tellegen, A. (1988). Development and validation of brief measures of positive and negative affect: The PANAS scales. Journal of Personality and Social Psychology, 54, 1063-1070.

Yates, M.S., Drewery, S. \& Murdoch-Eaton, D.G. (2002). Alternative learning environments: what do they contribute to professional development of medical students? Medical Teacher, 24(6), 609-615.

Table 1. Mood adjective checklist

Below are a number of words which describe moods. Please put a cross to indicate how much you have felt the way described, in the last 24 hours.

\begin{tabular}{l|l|l|l|l}
\hline & Not at all & A little & Quite a bit & Extremely \\
\hline Shaky & & & & \\
\hline Sluggish & & & & \\
\hline Resentful & & & & \\
\hline Nervous & & & & \\
\hline Weary & & & & \\
\hline Vigorous & & & & \\
\hline Hopeless & & & & \\
\hline Lively & & & & \\
\hline Guilty & & & & \\
\hline Tired & & & & \\
\hline Unhappy & & & & \\
\hline Tense & & & & \\
\hline Full of energy & & & & \\
\hline Active & & & & \\
\hline Worthless & & & & \\
\hline Miserable & & & & \\
\hline Worn out & & & & \\
\hline Discouraged & & & & \\
\hline Spiteful & & & & \\
\hline Depressed & & & & \\
\hline On edge & & & & \\
\hline Angry & & & & \\
\hline Furious & & & & \\
\hline Helpless & & & & \\
\hline
\end{tabular}


Table 2. Key themes expressed by patients during object handling sessions, including frequencies.

\begin{tabular}{l|l}
\hline Theme & Frequency \\
\hline Enjoyment / Interest & 24 \\
\hline $\begin{array}{l}\text { Boredom that was suppressed by session / } \\
\text { Appreciation of enrichment activities }\end{array}$ & 11 \\
\hline $\begin{array}{l}\text { Boredom caused by session / Lack of interest in the } \\
\text { session }\end{array}$ & 18 \\
\hline Pain & 3 \\
\hline Positive descriptive terms (e.g. beautiful, pretty) & 8 \\
\hline
\end{tabular}



Please indicate how satisfied you feel overall with your life at the moment by putting a cross on the line between 0 and 100.



Please indicate how you feel about your health status at the moment by putting a cross on the line between 0 and 100 .

Figure 1. Generic visual analogue scales 